\title{
NOUVELLE
}

\section{Cellules cancéreuses, cellules étoilées et rigidité du milieu extracellulaire}

\section{Le cercle vicieux du développement des métastases hépatiques}

Molène Docq ${ }^{1}$, Boris Julien ${ }^{2}$

\author{
${ }^{1}$ MI Biologie Santé, Université Paris-Saclay, 91405 Orsay, \\ France. \\ ${ }^{2}$ Institut Curie, U1021/UMR3347, Université Paris-Saclay, \\ 91405 Orsay, France. \\ molene.docq@universite-paris-saclay.fr \\ boris.julien@universite-paris-saclay.fr
}

> Le foie est un organe propice au développement des métastases de nombreux cancers. Cette funeste propriété est la conséquence d'une série d'interactions entre les cellules tumorales et le microenvironnement hépatique aboutissant à la constitution d'une niche favorable à leur implantation et à leur développement [1]. Les cellules stellaires hépatiques (HSC) jouent un rôle primordial dans ces interactions. Elles ont pour rôle physiologique de soutenir les cellules du parenchyme dans leurs fonctions et de stocker la vitamine A. Mais à la suite de lésions hépatiques, elles sont activées en myofibroblastes (MF) qui participent alors à la réparation tissulaire en remodelant la matrice extracel- lulaire (MEC) et produisent un ensemble de médiateurs agissant sur les autres cellules ou de manière endocrine. Or, les cellules tumorales détournent ces propriétés à leur avantage en activant les HSC en MF qui déposent massivement de la MEC et contribuent alors à la constitution d'une niche favorable au développement métastatique [2, 3] (Figure 1). II est désormais bien établi que la rigidité de la MEC, qui dépend de sa quantité et de sa composition, promeut la croissance tumorale qui, elle-même, exerce une pression mécanique sur son environnement [4]. Cependant, les mécanismes de rétrocontrôle qu'exercerait la rigidité du milieu extracellulaire sur les HSC sont encore à élucider. Dans un article récent,
Dou et al. ont montré que la rigidité de l'environnement des HSC favorisent leurs propriétés pro-tumorales selon une boucle d'amplification mécanosensible impliquant l'histone acétyltransférase p300 [5].

Plus l'environnement est rigide, plus les HSC s'activent : rôle de la signalisation RhoA/Akt/p300

Pour mettre en évidence le lien entre rigidité environnementale et activation des HSC, les auteurs ont cultivé des HSC (cultures de cellules primaires murines et humaines et de lignées humaines) sur des hydrogels de polyacrylamide de rigidité croissante. Ils ont observé que plus le milieu est rigide, plus les 


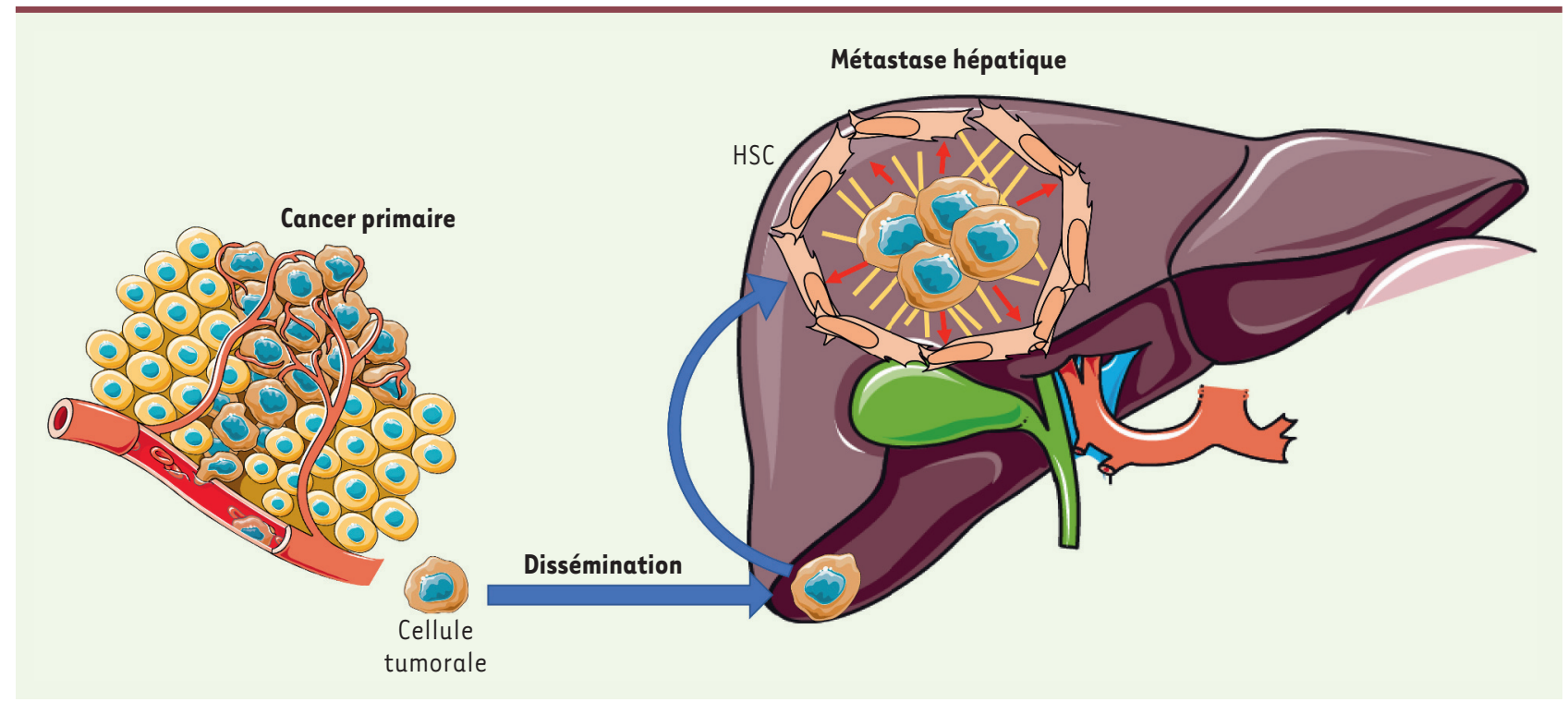

Figure 1. Les étapes à l'origine du développement d'une métastase hépatique. Les cellules tumorales sont susceptibles de s'échapper de la tumeur primaire et de se disséminer dans l'organisme. Le foie est un organe privilégié de leur implantation. Les cellules tumorales y recrutent et y exploitent les propriétés des cellules du micro-environnement hépatique, telles que les cellules stellaires hépatiques (HSC) qui se différencient alors en myofibroblastes et contribuent à la mise en place d'un environnement rigide (traits jaunes) dont le stress mécanique (flèches rouges) favorise le développement d’une métastase (cette figure a été créée en utilisant les modèles «Servier Medical Art »-https://smart.servier.com).

HSC expriment I' $\alpha$-SMA ( $\alpha$-smooth muscle actin) et le CTGF (connective tissue growth factor), deux marqueurs de différenciation myofibroblastique. Cette activation s'accompagne d'une translocation dans le noyau de l'histone acétyltransférase $\mathrm{p} 300$, qui régule de nombreux gènes impliqués dans la prolifération, l'apoptose et la différenciation cellulaire, en acétylant les histones et les facteurs de transcription [6]. II a notamment été montré que lors de la sclérodermie, pathologie provoquant une accumulation excessive de matrice dans les tissus, p300 contrôle la synthèse par les fibroblastes du collagène de type I, principal facteur de rigidité matricielle [7]. Dou et al. ont montré que l'invalidation du gène codant $p 300$ par des techniques moléculaires ainsi que l'inhibition pharmacologique de la fonction de la protéine, réduisent l'effet de la rigidité sur l'expression de l' $\alpha$-SMA dans des cultures primaires de HSC [5]. Ils mettent ainsi en évidence pour la première fois l'implication de p300 dans l'activation des HSC par la rigidité de leur environnement.
La translocation de p300 dans le noyau, où se situent ses substrats, dépend de modifications post-transcriptionnelles, telles que la phosphorylation. Selon les auteurs, deux protéines impliquées dans la transduction du signal mécanique conduisent à la translocation de p300: la petite protéine G RhoA (Ras homolog gene family, member $A$ ), un des relais de la signalisation intracellulaire des intégrines, et la protéine kinase AKT (protéine kinase B). Par des jeux d'inhibition et d'activation de ces protéines (inhibiteurs pharmacologiques de RhoA et AKT, surexpression de RhoA constitutivement activée ou de p300 mutée sur son site de phosphorylation par Akt) en utilisant leur modèle de culture en gel de polyacrylamide, les auteurs ont caractérisé finement les différentes étapes de cette signalisation (Figure 2): la rigidité du milieu active RhoA qui stimule alors la phosphorylation de Akt; ainsi activée, Akt phosphoryle $\mathrm{p} 300$, ce qui inhibe sa dégradation par le protéasome et favorise sa translocation dans le noyau où p300 exerce son activité biologique.
L’axe rigidité/p300 : voie pro-tumorale et pro-métastatique dans les MFs associés aux tumeurs

Cette voie de signalisation ayant été mise en évidence ex vivo, une question s'impose: est-elle pertinente in vivo? Pour répondre à cette question, les auteurs ont utilisé un modèle de souris CollAlCre/P300loxP présentant une invalidation du gène codant $p 300$ spécifiquement dans les MF (Collal est une protéine principalement exprimée dans les cellules fibrogéniques). Ils ont montré qu'en absence de p300 dans les MF, l'injection de cellules cancéreuses colorectales humaines génère moins de métastases hépatiques que dans des souris sauvages et que les MF associés à ces métastases étaient moins activés. Cette démonstration du lien entre cet axe mécanosensible et le développement tumoral est renforcée par l'utilisation de modèles murins de co-implantation sous-cutanée de cellules cancéreuses colorectales humaines avec des milieux conditionnés de HSC ayant été cultivées dans des conditions de rigidités différentes et exprimant ou non p300. Les 


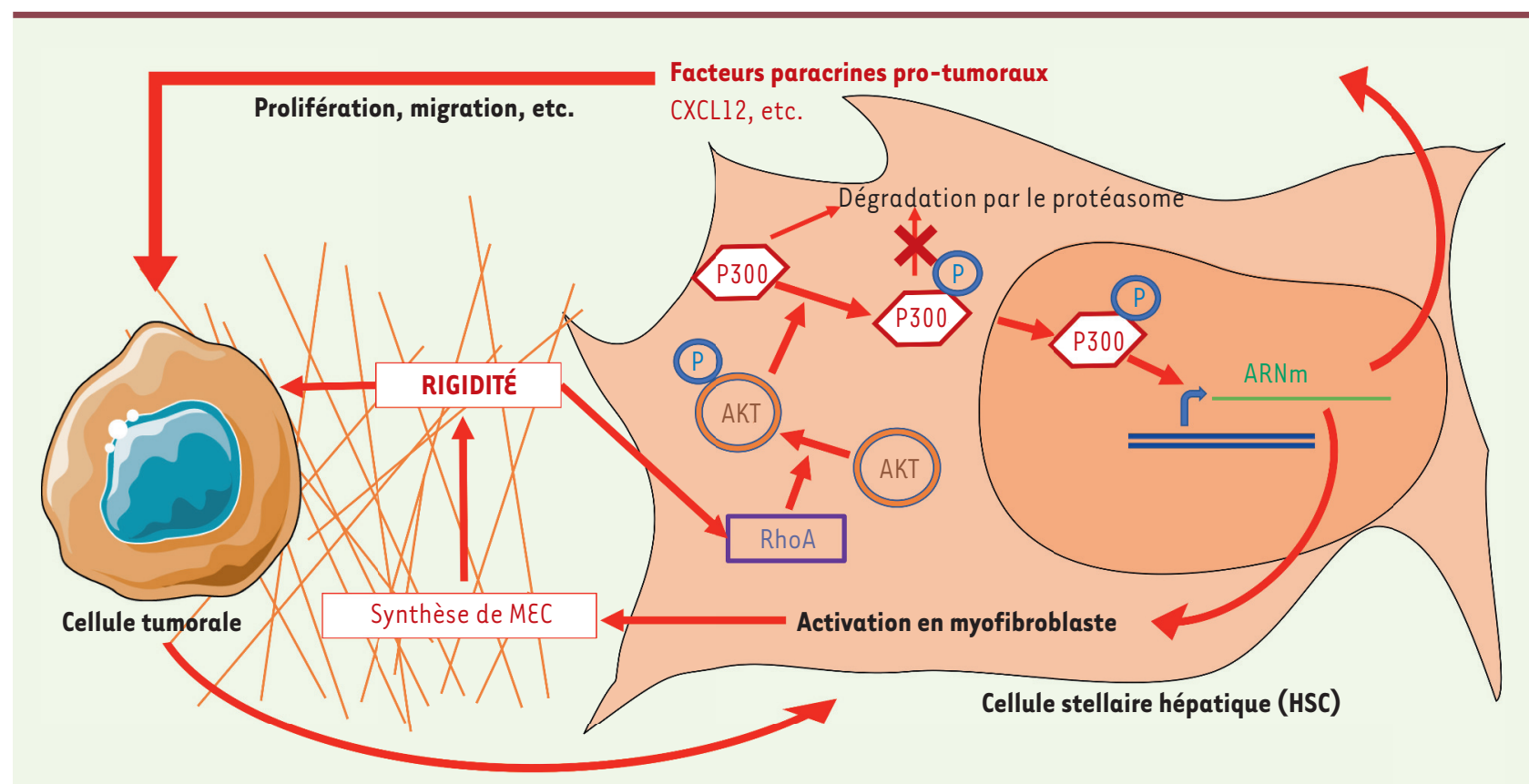

Recrute et active

Figure 2. Boucle d'amplification mécanosensible p300-dépendante favorisant le développement tumoral. La rigidité de l'environnement péritumoral est issue de l'action combinée de la croissance de la tumeur et de l'accumulation de matrice extracellulaire (MEC, traits oranges). Cette rigidité influence la biologie des cellules stellaires hépatiques (HSC) selon une boucle d'amplification mécanosensible dans laquelle p300 est relocalisée dans le noyau une fois phosphorylée par AKT, elle-même activée par RhoA. Ceci aboutit à la différenciation des HSC en myofibroblastes qui produisent de la matrice extracellulaire, et à l'expression de facteurs, tels CXCL12, favorisant la croissance des tumeurs (cette figure a été créée en utilisant les modèles Servier Medical Art - https://smart.servier.com).

milieux conditionnés de HSC cultivées sur un support rigide favorisent beaucoup plus le développement des tumeurs que ceux de cellules cultivées sur un support lâche, mais cette différence est perdue si les HSC sont déficientes pour p300. Ainsi, les auteurs ont émis I'hypothèse que les HSC produisent des facteurs pro-tumoraux et pro-métastatiques, dont la sécrétion est potentialisée par l'axe p300-rigidité.

Afin d'identifier les facteurs pro-tumoraux associés à l'activation des HSC, les auteurs ont comparé par RNA-seq (séquençage des transcrits) les ARNm des HSC cultivées sur support rigide ou lâche. Ils ont mis en évidence que l'axe rigidité-p300 module l'expression de nombreux gènes impliqués dans la différenciation en $M F$, mais aussi de gènes codant des médiateurs intercellulaires pro-tumoraux, tels le CTGF (connective tissue growth factor) ou la chimiokine
CXCL12 (C-X-C motif chemokine 12), connue pour réguler l'implantation des métastases dans le foie [8]. En présence d'un milieu rigide, l'expression protéique de CXCL12 est augmentée dans les MF et les histones de son promoteur sont acétylées, comme le démontrent des expériences d'immunoprécipitation des histones acétylées suivies de l'identification du promoteur de CXCL12 par qPCR. Ces observations renforcent encore la démonstration du lien entre l'activation des HSC en MF induite par la rigidité du support et l'histone acétylase p300.

\section{p300 et HSC : au-delà de la prise} en charge des métastases hépatiques Dou et al. ont montré pour la première fois que la rigidité du milieu extracellulaire induit la différenciation des cellules stellaires hépatiques en myofibroblastes selon une boucle d'amplifi- cation mécanosensible mettant en jeu p300 et ses partenaires AKT et RhoA [5]. Cette activation mécanique des HSC qui dépend de p300 contribue à l'implantation et au développement de métastases dans le foie (Figure 2). Plusieurs de leurs résultats suggèrent que cette démonstration pourrait être validée en pathologie humaine. D'une part, leurs observations ex vivo ont été effectuées sur des HSC humaines. D'autre part, dans des biopsies de patients ayant des métastases hépatiques de cancers colorectaux, le stroma tumoral, qui est riche en myofibroblastes, est plus rigide que les tissus environnants et surexprime $\mathrm{p} 300$. Ce co-facteur de transcription pourrait donc être l'objet d'un ciblage thérapeutique visant à diminuer les propriétés pro-métastatiques du microenvironnement hépatique. Néanmoins, cette découverte pourrait avoir des retombées plus larges. En effet, 
Dou et al. ont également montré dans ces travaux que p300 régulait aussi la fibrose hépatique dont le stade ultime, la cirrhose, est responsable d'une mortalité importante (environ 10000 morts par an en France) [5]. De plus, les MF sont la principale source des fibroblastes associés au cancer (CAF) dans le foie [3]. Or les CAF sont présents dans le stroma de la plupart des tumeurs solides et favorisent leur développement notamment en provoquant un stress mécanique [9]. Ainsi, l'axe rigidité/p300/activation des cellules fibrogéniques mis en évidence par Dou et al participerait au développement de différents types de cancers associés à l'activation de MF et au dépôt de MEC. Le profil d'expression de p300 et ses effets pléïotropes exposeraient cependant un patient traité avec un inhibiteur à de nombreux effets secondaires. II serait donc nécessaire d'envisager un ciblage des cellules fibrogéniques, ce qui constitue un défi scientifique et médical depuis de nombreuses années [10]. $\diamond$

Cancer cell, stellate cells and stiffness: the vicious circle of hepatic metastasis

\section{LIENS D'INTÉRÊT}

Les auteurs déclarent n'avoir aucun lien d'intérêt concernant les données publiées dans cet article.

\section{RÉFÉRENCES}

1. Vidal-Vanaclocha F. The prometastatic microenvironment of the liver. Cancer Microenviron $2008 ; 1: 113-29$.

2. Lu P, Weaver VM, Werb Z. The extracellular matrix: a dynamic niche in cancer progression. J Cell Biol 2011 ; $96: 395-406$

3. Kang N, Gores GJ, Shah VH. Hepatic stellate cells: partners in crime for liver metastases? Hepatology $2011 ; 54: 707-13$.
4. Schrader J, Gordon-Walker $\Pi$, Aucott RL, et al. Matrix stiffness modulates proliferation, chemotherapeutic response, and dormancy in hepatocellular carcinoma cells. Hepatology $2011 ; 53$ : 1192-205.

5. Dou C, Liu Z, Tu K, et al. P300 Acetyltransferase mediates stiffness-induced activation of hepatic stellate cells Into tumor-promoting myofibroblasts. Gastroenterology 2018 ; 154 : 2209-21.el4.

6. Chan HM, La Thangue NB. p300/CBP proteins: HATs for transcriptional bridges and scaffolds. J Cell Sci 2001 $114: 2363-73$.

7. Ghosh AK, Bhattacharyya S, Lafyatis R, et al. p300 is elevated in systemic sclerosis and its expression is positively regulated by TGF- $\beta$ : epigenetic feedforward amplification of fibrosis. J Invest Dermatol 2013 ; 133 : 1302-10.

8. Sakai N, Yoshidome H, Shida T, et al. CXCR4/ CXCL12 expression profile is associated with tumor microenvironment and clinical outcome of liver metastases of colorectal cancer. Clin Exp Metastasis 2012 ; 29 : 101-10.

9. Nissen NI, Karsdal M, Willumsen N. Collagens and cancer associated fibroblasts in the reactive stroma and its relation to cancer biology. J Exp Clin Cancer Res 2019; $38: 115$.

10. Beljaars L, Meijer DK, Poelstra K. Targeting hepatic stellate cells for cell-specific treatment of liver fibrosis. Front Biosci 2002 ; 7 : e214-22.

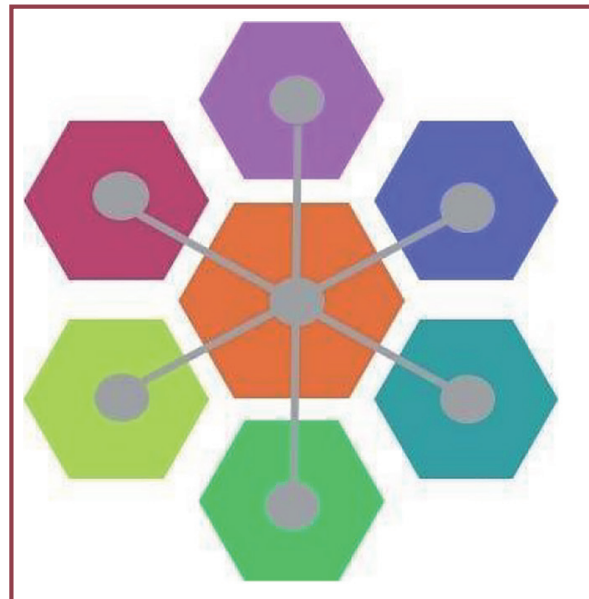

\section{Global Registry for COL6-related dystrophies}

\section{Registre global des dystrophies liées au collagène de type VI}

S'inscrire sur : www.collagenb.org

Ou contactez-nous par e-mail à l'adresse : collagenbregistry@ncl.ac.uk

\section{La traduction française sera bientôt disponible sur le site web.}
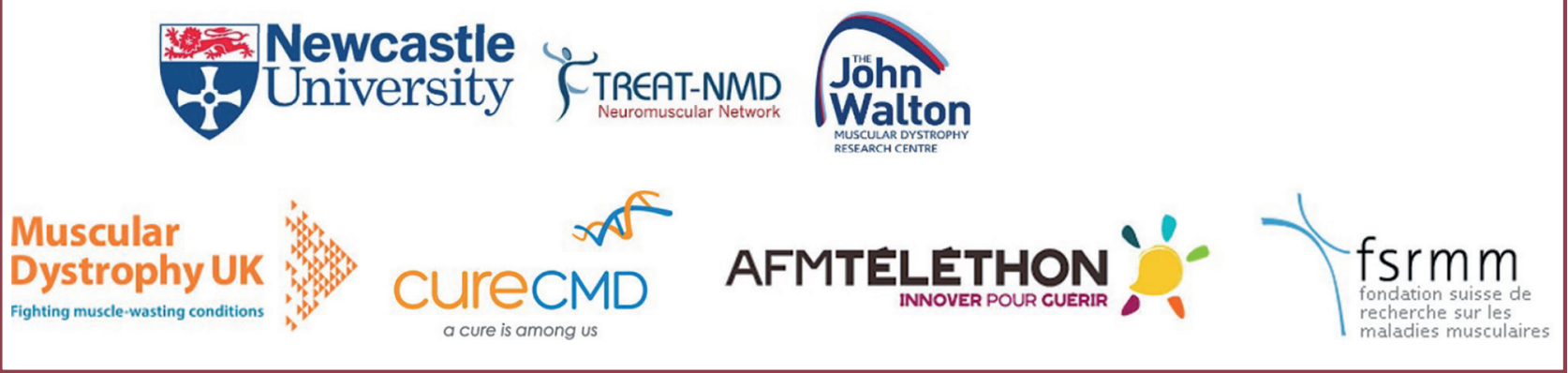